\title{
European regulator appeals against order stopping it from releasing clinical study documents
}

Clarification-In this News story by Zosia Kmietowicz (BMJ 2013;347:f4728, doi:10.1136/bmj.f4728), we said that the BMJ's application to intervene in the case in support of the European Medicines Agency has been accepted by the European court.
We would like to point out that this application is a joint initiative between the BMJ and the BMA.

Cite this as: BMJ 2013;347:f4867

๑ BMJ Publishing Group Ltd 2013 